\title{
Trace Metal Contamination in Water from Abandoned Mining and Non-Mining areas in the Northern Parts of the Ashanti Gold Belt, Ghana
}

\author{
C. Tay ${ }^{1 *}$ and F. W. Y. Momade ${ }^{2}$ \\ ${ }^{1}$ CSIR-Water Research Institute, P. O. Box AH 38, Achimota-Accra, Ghana \\ ${ }^{2}$ Department of Material Engineering, Kwame Nkrumah University of Science and Tehcnology, \\ Kumasi, Ghana \\ *Corresponding author
}

\begin{abstract}
A survey was conducted on the levels of trace metals ( $\mathrm{Ni}, \mathrm{Cu}, \mathrm{Hg}, \mathrm{Pb}, \mathrm{Mn}, \mathrm{Fe}, \mathrm{Zn}, \mathrm{Cr}, \mathrm{Cd}, \mathrm{Mg}$ and $\mathrm{Ca}$ ) in ground and surface water sources from the northern parts of the Ashanti gold belt. Water samples were collected from 67 boreholes, 24 wells, and 10 streams during dry and wet seasons for trace metal analyses using Philips PU 9200 atomic absorption spectrophotometer. The main objective was to determine whether trace metal contamination in the study area was as a result of mining or geochemical and biochemical processes within the aquifer. The results showed that ground water $(p \mathrm{H}$ range 4.09-7.29 and mean $5.87 p \mathrm{H}$ units) was slightly acidic (low $p \mathrm{H})$ than surface water $(p \mathrm{H}$ range 5.81-7.74 and mean $7.12 \mathrm{pH}$ units). The ground waters (with conductivity range 96-1553 $\mathrm{SS} / \mathrm{cm}$ and mean 407.7 $\mu \mathrm{S} / \mathrm{cm}$ ) in the study area were also more mineralized than surface waters (with conductivity range 113-540 $\mu \mathrm{S} / \mathrm{cm}$ and mean $323.30 \mu \mathrm{S} / \mathrm{cm})$. Correlations between the trace metals revealed expected process-based relationship between $\mathrm{Mg}^{2+}$ and $\mathrm{Ca}^{2+}(r=0.76)$, derived mainly from the geochemical and biochemical processes within the aquifer. However, there were also other strong linear relationships between trace metals, such as $\mathrm{Fe}^{2+}$ and $\mathrm{Ca}^{2+}(r=0.61) ; \mathrm{Mg}^{2+}$ and $\mathrm{Mn}^{2+}$ $(r=0.72), \mathrm{Hg}^{2+}$ and $\mathrm{Mn}^{2+}(r=0.61)$, that are not normally expected to be linked in terms of processes. All the well and stream water sources had one or more trace metal level outside acceptable limits set by the World Health Organization for drinking water, and only three borehole water samples at Konongo (Abronikrom), Ntronang (Ahenbronoso) and Bomfa (Nkubem) were safe for human consumption.
\end{abstract}

\section{Introduction}

Water pollution, caused either by mining activities or natural geochemical and biochemical processes (such as the oxidation of pyrite and arsenopyrite in an aquifer), is a significant problem in several countries in the world. In Tanzania, for example, $78 \%$ of water samples analysed in the Lake Victoria goldfields contained mercury in concentrations significantly above the drinking water standard of $1 \mathrm{~g} / \mathrm{l}$ (Mpendazoe, 1996). The presence of trace metals in rivers and river sediments in small-scale mining areas in Tanzania is a threat to both the environment and to human health.

The contamination of water resources either by mining activities or natural geochemical and biochemical processes in Mozambique is another example. The Mozoe, Luenha, Revue and Zambezi rivers flow through Mozambique to the India Ocean, from Zimbabwe, Zambia and Malawi, where the use of mercury is a common practice, and contribute to water contamination downstream (RT1 ITDG, 2001). The presence of excessive heavy metals and other components of mining effluents have widespread toxic effects on living organisms. In addition, these substances also reduce the fitness of water for human consumption and other uses.

In Ghana, most of the mining companies are located on the Ashanti belt. Conse-quently, mining activities have, over the years, impacted on water resources within the belt through inadequate management of tailings and effluents from processing plants, exposure of mined surfaces leading to acid mine drainage, and from the use of mercury by the small-scale alluvial workings (Anon., 1999). Very often, rivers or streams that at one time had served as sources of drinking water for the communities have been declared unsafe and users advised to stay away (Anon., 1999). Natural geochemical and biochemical processes in aquifers may also lead to 
changes in the characteristics of the waters in contact with the aquifer. The result is the change in the characteristics of both ground and surface waters which serve as sources of water supply to the inhabitants of these regions.

The Ashanti gold belt does not only serve as a residential area to mining companies but also to a large percentage of the population in the Ashanti Region of Ghana, which is mainly engaged in farming and other activities outside mining. For many of them, ground (boreholes and wells) and surface (streams and rivers) waters are the sources of water for domestic uses. However, effluents from the mining industry may pollute some of these water bodies. Geochemical and biochemical processes in exposed outcrops may also lead to increased acidity of the water in contact, increasing the solubility of some trace metal compo-nents and, therefore, rendering some of these water sources unusable. It is, thus, essential to identify the water resources within the Ashanti gold belt and determine the actual sources of impact on the quality of water bodies in relation to the geochemistry or mining activity. A proper understanding of contaminant inputs and impact is a necessary tool for sustainability, ensuring that future generations will be able to rely on the water bodies and land in the belt.

The literature indicates that some of these issues have been studied by some investigators in Ghana (Sarkodie et al., 1997; Amonoo-Niezer \& Amenkor, 1993; Golow et al., 1996; Carboo \& Sarfo-Armah, 1997). Many of these studies, however, have been concentrated in areas where mining operations are ongoing. In some cases, only a few parameters have been examined. Preliminary investigations conducted by the Institute of Mining and Minerals Engineer-ing, KNUST, Kumasi have revealed that some of the parameters relating to water quality, such as the $p \mathrm{H}$, and some trace metals, such as nickel and manganese, of some streams originating from the belt, wells and boreholes, in certain areas of the belt and outside mining areas, are outside acceptable limits set by the World Health Organization (WHO) for drinking water (Hofkes, 1986). The study did not, however, cover all the trace metals of concern, such as mercury, chromium and lead arsenic, among others.

The paper seeks to assess the trace metal concentrations of the water resources in the northern parts of the Ashanti gold belt in order to determine the impact of mining activities on the quality of water bodies.

\section{Study area}

\section{Materials and methods}

The Ashanti gold belt is the most prominent of the gold belts in Ghana and stretches for over 200 $\mathrm{km}$ within the central parts of the country, from Akanko, near the coast, through Prestea and Obuasi to Konongo. This ore channel is the home of prominent gold mines, including the Abosso Goldfields Ltd, Goldfields Ghana Ltd, the Billiton Bogosu Gold Ltd, the Ashanti Goldfields Company Ltd, and the Anglogold Ashanti Bibiani Mine (Dzigbordi-Adjimah, 1988). The belt is situated in the Birimian and Tarkwaian systems. Fig. 1 presents the Ashanti gold belt and the sampling communities. 


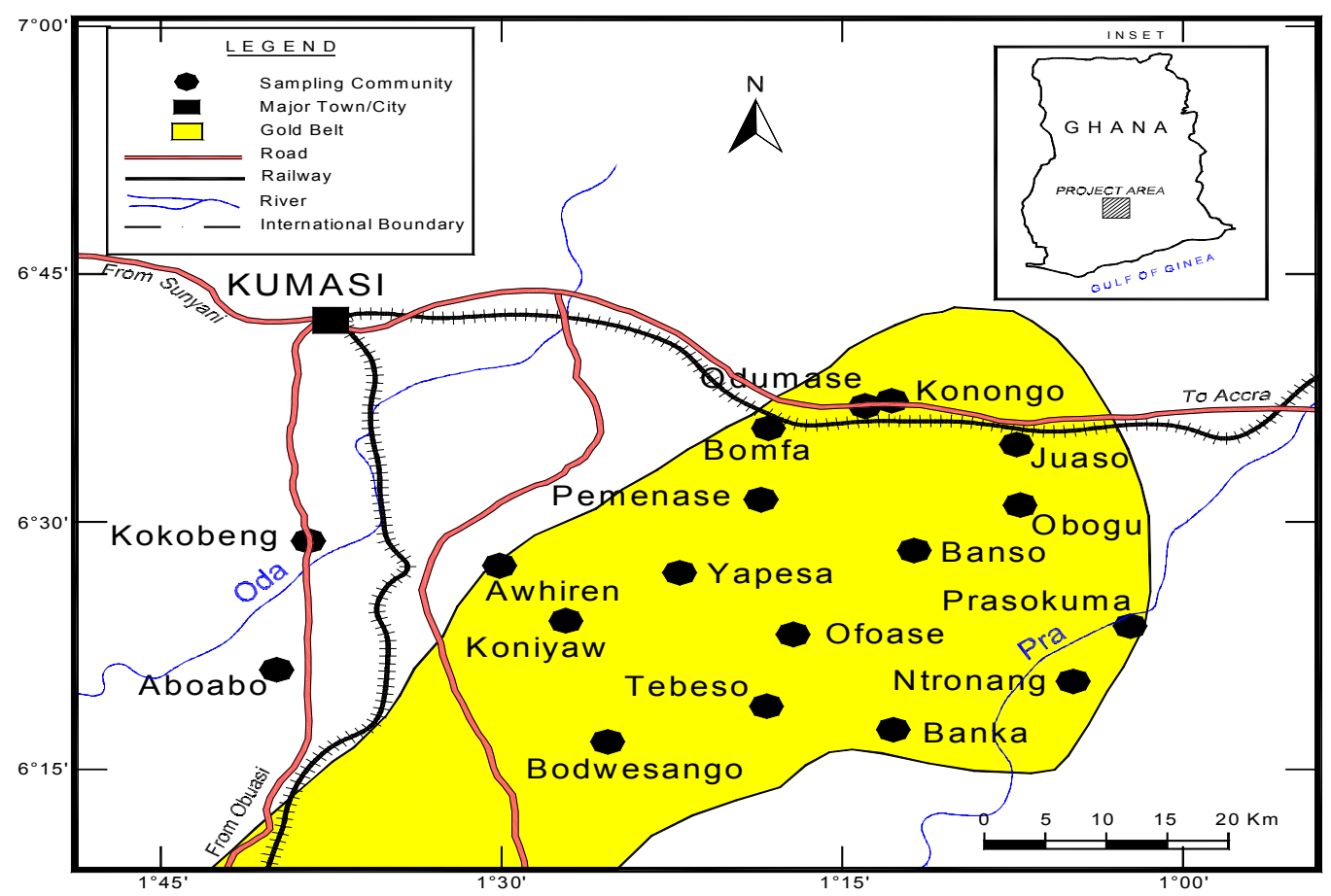

Fig. 1. The Ashanti gold belt and the sampling locations

\section{Birimian system}

The Birimian system is made up of sedimentary and volcanogenic sequences that have been metamorphosed to slates; phyllites, metagreywackes and meta-volcanic rocks, which are interbeded with metamorphosed pyroclastic members. The primary gold of the Birimian occurs with a black lustrous carbonaceous material loosely referred to as 'graphite' and with sulphides, copper and lead sulpho-salts (Dzigbordi-Adjimah, 1988). A greater quantity of this gold, however, comes from the primary lode-type deposits. These lodes are considered the parent source of the Banket and alluvial type gold (Junner, 1935).

\section{Ore geology}

Ore mineralization in the country rock. Ore types of the country rock which characterizes the ore mineralization of the Ashanti gold belt consist of rutile $\left(\mathrm{TiO}_{2}\right)$, arsenopyrite ( $\left.\mathrm{FeAsS}\right)$ and pyrite $\left(\mathrm{FeS}_{2}\right)$. Rutile which is formed from the alteration of "sandwich-type" magnetite/ilmenite (a primary constituent of the rocks) occurs as disseminated grains in the country rock and is often aligned parallel to the foliation planes (Amanor, 1979). Rutile also appears to pre-date the sulphides and it is disseminated in all the ores. Arsenopyrite and pyrrhotite are the primary sulphides in the wall-rocks, but pyrite is predominantly of secondary origin (namely, from the transformation of pyrrhotite). Visible gold is rare in the wall rocks but submicroscopic gold occurs in the sulphides. When such mineralizations reach ore grade they are generally referred to as "disseminated sulphide ore" (Amanor, 1979).

Paragenesis of the quartz ore-bodies. Sulphides and sulphosalts are the main ore minerals of the quartz lenses. About $20 \%$ of the gold is native and contains about $10 \%$ silver; such "free" gold is liberated by grinding and gravity separation (Afenya, 1976). The bulk of the gold is, however, intimately associated with one or other of the following sulphides: pyrite $\left(\mathrm{FeS}_{2}\right)$, marcasite $\left(\mathrm{FeS}_{2}\right)$, arsenopyrite $(\mathrm{FeAsS})$, sphalerite $(\mathrm{ZnS})$, chalcopyrite $\left(\mathrm{CuFeS}_{2}\right)$, bornite $\left(\mathrm{Cu}_{5} \mathrm{FeS}_{4}\right)$ and galena $(\mathrm{PbS})$. Alabandite $(\mathrm{MnS})$ was reported at Obuasi (Amanor, 1979). The 
sulphosalts of the ore include tetrahedrite $\left(\mathrm{Cu}_{12} \mathrm{Sb}_{4} \mathrm{~S}_{13}\right)$, boumonite $\left(\mathrm{PbCuSb}_{3}\right) \mathrm{S}$, boulangerite $\left(\mathrm{Pb}_{5} \mathrm{Sb}_{4} \mathrm{~S}_{11}\right)$ and jamensonite $\left(\mathrm{Pb}_{4} \mathrm{FeSb}_{8} \mathrm{~S}_{24}\right)$. Both magmatic crystallization differentiation as well as lateral secretion theories (Boyle, 1959) have been used to explain the origin of the deposits.

Junner (1932), Cooper (1934) and P. J. Uebel (1986, personal communication) believe that the ore-bodies together with the ore constituents represent an end-product of a through-going magmatic hydrothermal solution, which originated from a sub-apexing granitic pluton at depth. They associate the gold mineralization with the Dixcove (or Bondoku) granite, which intrude the Birimiam. Ntiamoah-Agyakwa $(1974,1979)$ proposed that the quartz ore-bodies are metamorphosed cherty horizons and that the ore constituents represent remobilized syngenetically introduced material that was concentrated laterally into dilatant fissures during tectonism. It could be inferred from the literature, therefore, that during weathering and leaching processes, various cations and anions will be introduced into water bodies and, consequently, the quality of these water bodies will be altered.

\section{Data analyses}

Water samples were collected from boreholes, wells and stream sources during the dry season (February 2000) and wet season (June 2000). Sixty-seven boreholes, 24 wells and 10 streams were sampled. Sampling protocols described by Claasen (1982) and Barcelona et al. (1985) were strictly adhered to during sample collection. Samples were collected using 4-litre acid-washed polypropylene containers. Bore-holes were sampled only after pumping for sometime; wells and stream sources were sampled a depth below the surface. All samples were stored in a refrigerator at a temperature of $<4{ }^{\circ} \mathrm{C}$ and analysed within 1 week. Temperature, $p \mathrm{H}$ and electrical conductivity were measured in situ using WTW-Multiline P4 Universal meter.

For the determination of trace metals, $10 \mathrm{ml}$ of the sample was pipetted into a Teflon crucible to which $1 \mathrm{ml}$ of nitric acid $\left(\mathrm{HNO}_{3}\right)$ was added to preserve the water by preventing it from any possible reactions before analysis. The mixture was heated in a pressure container at $120{ }^{\circ} \mathrm{C}$ for 4 $\mathrm{h}$. The resulting solution was analysed for trace metals at the Institute of Mining and Mineral Engineering (IMME) Laboratory of the Kwame Nkrumah University of Science and Technology, Kumasi, using Philips PU 9200 atomic absorption spectrophotometer. All the trace metals were determined by flame atomization.

\section{Results and discussion}

The mean concentrations of trace metals and the WHO guideline values are presented in Table 1 and the wet and dry seasons range values are presented in Table 2.

TABLE 1

Mean parameters of the study area for February and June 2000

\begin{tabular}{|c|c|c|c|c|c|c|c|c|c|c|}
\hline Sample & Location & Temp. & $\mathrm{p} H$ & Cond. & $\mathrm{Ni}$ & & $\mathrm{Cu}$ & $H g$ & $\mathrm{~Pb}$ & $M n$ \\
\hline $\begin{array}{l}\mathrm{Cd} \\
\mathrm{No}\end{array}$ & $M g$ & $\begin{array}{l}\mathrm{Zn} \\
{ }^{\circ} \mathrm{C}\end{array}$ & $\mathrm{Ca}$ & $\begin{array}{r}\mathrm{Cr} \\
\mathrm{mS} / \mathrm{cm}\end{array}$ & $m g / l$ & & $m g / l$ & $m g / l$ & $m g / l$ & $m g / l$ \\
\hline$m g / l$ & $m g / l$ & $m g / l$ & $m g / l$ & $m g / l$ & & & & & & \\
\hline B1 & Odumasi market & 27.5 & 5.08 & 830 & 0.1 & 0.01 & 0.22 & 0.04 & 0.34 & 0.3 \\
\hline 0.005 & 7.98 & 0.15 & 14.66 & 0.01 & & & & & & \\
\hline B2 & Odumasi (Zongo) & 29 & 5.56 & 380.5 & 0.02 & 0.01 & 0.14 & 0.01 & 0.33 & 0.03 \\
\hline 0.001 & 3.08 & 0.04 & 2.16 & 0.07 & & & & & & \\
\hline B3 & Konongo-Odumasi sec. Sch. & 28 & 6.6 & 307.5 & 0.03 & 0.01 & 0.16 & 0.03 & 0.08 & 0.13 \\
\hline 0.001 & 1.06 & 1 & 2.07 & 0.01 & & & & & & \\
\hline B4 & Konongo (Abronikrom) & 29 & 6.37 & 224.5 & 0.02 & 0.01 & 0.001 & 0.01 & 0.01 & 0.04 \\
\hline 0.003 & 2.71 & 1 & 1.51 & 0.01 & & & & & & \\
\hline B5 & Juaso Dist.Hospital & 29.5 & 5.89 & 856.5 & 0.07 & 0.04 & 0.03 & 0.01 & 0.08 & 0.04 \\
\hline
\end{tabular}




\begin{tabular}{|c|c|c|c|c|c|c|c|c|c|c|c|}
\hline 0.001 & 0.76 & 0.02 & 0.41 & 0.01 & & & & & & & \\
\hline B6 & Obogu (Savior Church & 29.5 & 7.05 & 449.5 & 0.05 & 0.01 & 0.02 & 0.01 & 0.01 & 0.05 & \\
\hline 0.005 & 3.62 & 1 & 4.01 & 0.02 & & & & & & & \\
\hline & Mission) A & & & & & & & & & & \\
\hline B7 & Obogu (Savior Church & 29 & 6.59 & 832 & 0.07 & 0.02 & 0.33 & 0.04 & 0.01 & 0.05 & \\
\hline 0.008 & 8.96 & 1 & 28.41 & 0.01 & & & & & & & \\
\hline & Mission) $\mathrm{B}$ & & & & & & & & & & \\
\hline B8 & Banso (Anikyeasa) A & 28.5 & 5.58 & 383 & 0.01 & 0.03 & 0.1 & 0.02 & 0.03 & 0.03 & \\
\hline 0.001 & 6.71 & 0.01 & 5.52 & 0.01 & & & & & & & \\
\hline B9 & Banso (Anikyeasa) B & 28.3 & 5.67 & 379 & 0.01 & 0.03 & 0.001 & 0.03 & 0.1 & 0.02 & \\
\hline 0.001 & 2.21 & 1 & 3.1 & 0.02 & & & & & & & \\
\hline B10 & Ofoase (Koforidua) & 29.3 & 4.57 & 229.5 & 0.14 & 0.06 & 0.24 & 0.03 & 0.01 & 0.03 & \\
\hline 0.001 & 1.1 & 0.11 & 1.24 & 0.03 & & & & & & & \\
\hline B11 & Ofoase (kubasi) & 29.5 & 4.64 & 232.5 & 0.07 & 0.11 & 0.001 & 0.03 & 0.06 & 0.02 & \\
\hline 0.024 & 1.13 & 0.48 & 1.54 & 0.02 & & & & & & & \\
\hline B12 & Tebeso No.1 A & 29 & 6.43 & 825 & 0.08 & 0.02 & 0.51 & 0.04 & 0.56 & 0.01 & \\
\hline 0.019 & 11.64 & 0.03 & 51.99 & 0.03 & & & & & & & \\
\hline B13 & Tebeso No.1 B & 29.2 & 6.23 & 451.5 & 0.05 & 0.02 & 0.42 & 0.06 & 0.34 & 0.06 & \\
\hline 0.017 & 10.82 & 0.01 & 17.83 & 0.01 & & & & & & & \\
\hline B14 & Banka Health Centre & 27 & 5.87 & 136.5 & 0.01 & 0.03 & 0.1 & 0.05 & 0.07 & 0.09 & \\
\hline 0.011 & 1.8 & 0.01 & 0.82 & 0.35 & & & & & & & \\
\hline B15 & Ntronong (Ahenbronoso) & 28 & 6.7 & 528.5 & 0.01 & 0.02 & 0.001 & 0.01 & 0.17 & 0.12 & \\
\hline 0.001 & 6.49 & 1 & 0.82 & 0.01 & & & & & & & \\
\hline B16 & Prasokuma B & 28 & 5.38 & 428 & 0.01 & 0.01 & 0.16 & 0.01 & 0.12 & 0.02 & 0.0 \\
\hline 2.3 & 0.02 & 2.72 & 0.01 & & & & & & & & \\
\hline B17 & Prasokuma A & 28 & 5.47 & 368 & 0.01 & 0.02 & 0.26 & 0.02 & 0.12 & 0.03 & \\
\hline 0.001 & 5.51 & 0.02 & 4.29 & 0.01 & & & & & & & \\
\hline B18 & Prasokuma C & 28 & 6.99 & 472.5 & 0.1 & 0.02 & 0.2 & 0.03 & 0.55 & 0.02 & \\
\hline 0.001 & 3.89 & 0.02 & 4.29 & 0.01 & & & & & & & \\
\hline B19 & Bomfa (Zongo) & 28.5 & 6.04 & 319 & 0.05 & 0.01 & 0.02 & 0.02 & 0.04 & 0.06 & \\
\hline 0.003 & 3.19 & 0.01 & 1.23 & 0.01 & & & & & & & \\
\hline B20 & Bomfa (Nkubem) & 28.5 & 6.27 & 540 & 0.05 & 0.01 & 0.001 & 0.02 & 0.04 & 0.02 & \\
\hline 0.003 & 5.65 & 1 & 2.18 & 0.01 & & & & & & & \\
\hline B21 & Bomfa (Ahenbrono) & 28 & 6.3 & 249.5 & 0.03 & 0.01 & 0.11 & 0.02 & 0.02 & 0.03 & \\
\hline 0.001 & 1.78 & 1 & 0.71 & 0.01 & & & & & & & \\
\hline B22 & Bomfa (Pampasu) & 28 & 6.19 & 238 & 0.01 & 0.02 & 0.06 & 0.01 & 0.1 & 0.01 & \\
\hline 0.001 & 1.33 & 1 & 0.58 & 0.01 & & & & & & & \\
\hline B23 & Pemenase (j.s.s mission) & 29 & 6.95 & 657.5 & 0.11 & 0.01 & 0.52 & 0.04 & 0.34 & 0.14 & \\
\hline 0.001 & 9.46 & 1 & 20.53 & 0.04 & & & & & & & \\
\hline B24 & Yapesa No. 1 & 27.5 & 7.12 & 553.5 & 0.05 & 0.01 & 0.13 & 0.01 & 0.25 & 0.67 & \\
\hline 0.004 & 13.07 & 1 & 52.14 & 0.01 & & & & & & & \\
\hline B25 & Ahwiren (Domiabra) A & 29 & 6.06 & 364 & 0.06 & 0.03 & 0.12 & 0.04 & 0.04 & 0.02 & \\
\hline 0.001 & 4.46 & 0.01 & 1.96 & 0.01 & & & & & & & \\
\hline B26 & Ahwiren (Domiabra) B & 28.5 & 5.99 & 558 & 0.01 & 0.05 & 0.52 & 0.04 & 0.75 & 0.05 & \\
\hline 0.003 & 1.86 & 0.05 & 1.86 & 0.01 & & & & & & & \\
\hline B27 & Koniyaw (Anafuo) & 29.5 & 5.88 & 311.5 & 0.01 & 0.02 & 0.001 & 1.03 & 0.04 & 0.02 & \\
\hline 0.004 & 2.13 & 0.01 & 1.53 & 0.01 & & & & & & & \\
\hline B28 & Koniyaw (Atifi) A & 28 & 6.43 & 302.5 & 0.13 & 0.01 & 0.19 & 0.01 & 0.22 & 0.71 & \\
\hline 0.001 & 3.7 & 1 & 2.44 & 0.03 & & & & & & & \\
\hline B29 & Koniyaw (Atifi) B & 28 & 5.95 & 534 & 0.01 & 0.01 & 0.13 & 0.04 & 0.02 & 0.02 & \\
\hline 0.001 & $4.33^{\circ}$ & 0.01 & 2.81 & 0.02 & & & & & & & \\
\hline B30 & Bodwesango town A & 28 & 4.5 & 218.5 & 0.05 & 0.04 & 0.23 & 0.09 & 0.1 & 2.12 & \\
\hline 0.004 & 0.9 & 0.02 & 1.66 & 0.01 & & & & & & & \\
\hline B31 & Aboabo NO. 2 A & 27.5 & 6.62 & 398 & 0.04 & 0.01 & 0.2 & 0.01 & 0.09 & 0.03 & \\
\hline 0.001 & 1.78 & 1 & 11.76 & 0.01 & & & & & & & \\
\hline B32 & Aboabo NO. 2 B & 28 & 4.61 & 357 & 0.01 & 0.01 & 0.2 & 0.02 & 0.2 & 0.02 & \\
\hline 0.004 & 1.09 & 0.03 & 1.74 & 0.01 & & & & & & & \\
\hline W1 & Odumasi (Zongo) A & 28.5 & 4.75 & 442.5 & 0.09 & 0.05 & 0.45 & 0.03 & 0.38 & 0.1 & \\
\hline 0.011 & 5.12 & 0.07 & 2.3 & 0.06 & & & & & & & \\
\hline
\end{tabular}




\begin{tabular}{|c|c|c|c|c|c|c|c|c|c|c|}
\hline W2 & Odumasi (Zongo) B & 28 & 4.71 & 402 & 0.01 & 0.02 & 0.6 & 0.03 & 0.24 & 0.16 \\
\hline 0.005 & 2.89 & 0.08 & 2.11 & 0.04 & & & & & & \\
\hline W3 & Konongo (Freetown) A & 27 & 5.75 & 398 & 0.09 & 0.01 & 0.03 & 0.04 & 0.06 & 0.06 \\
\hline 0.004 & 4.79 & 0.01 & 1.05 & 0.009 & & & & & & \\
\hline W4 & Konongo (Freetown) B & 29.5 & 5.42 & 714.5 & 0.02 & 0.02 & 0.09 & 0.02 & 0.1 & 0.05 \\
\hline 0.007 & 10.8 & 0.06 & 9.13 & 0.01 & & & & & & \\
\hline W5 & Konongo (Freetown) $\mathrm{C}$ & 29 & 6.49 & 640 & 0.09 & 0.02 & 0.001 & 0.03 & 0.05 & 0.15 \\
\hline 0.006 & 7.46 & 0.04 & 1 & 0.05 & & & & & & \\
\hline W6 & Juaso Y-junction A & 29.5 & 5.51 & 140.5 & 0.01 & 0.06 & 0.001 & 0.01 & 0.04 & 0.26 \\
\hline 0.007 & 1.93 & 1 & 0.94 & 0.04 & & & & & & \\
\hline W7 & Juaso Y-junction B & 29 & 5.27 & 142.5 & 0.09 & 0.01 & 0.001 & 0.01 & 0.03 & 0.17 \\
\hline 0.001 & 1.33 & 0.05 & 0.67 & 0.08 & & & & & & \\
\hline w8 & Obogu (Zongo) A & 29.5 & 6.51 & 268 & 0.01 & 0.02 & 0.001 & 0.02 & 0.06 & 0.08 \\
\hline 0.007 & 2.64 & 1 & 1.68 & 0.08 & & & & & & \\
\hline W9 & Obogu (Zongo) B & 28.5 & 5.04 & 246 & 0.13 & 0.04 & 0.23 & 0.01 & 0.23 & 0.26 \\
\hline 0.007 & 2.67 & 0.02 & 0.58 & 0.01 & & & & & & \\
\hline W10 & Obogu (Zongo) C & 28.5 & 5.1 & 678.5 & 0.06 & 0.03 & 0.29 & 0.01 & 0.1 & 0.08 \\
\hline 0.012 & 4.2 & 0.02 & 3.13 & 0.01 & & & & & & \\
\hline W11 & Bomfa (Penipa) B & 28 & 5.97 & 323 & 0.05 & 0.04 & 0.02 & 0.01 & 0.05 & 0.11 \\
\hline 0.001 & 2.35 & 1 & 0.97 & 0.01 & & & & & & \\
\hline W12 & Pemenase (Sikafoabantem) & 29 & 6.13 & 309.5 & 0.03 & 0.01 & 0.3 & 0.03 & 0.06 & 0.88 \\
\hline 0.001 & 5.42 & 1 & 0.93 & 0.01 & & & & & & \\
\hline $\mathrm{S} 1$ & Odumasi (outskirts) & 27 & 7.17 & 228.5 & 0.08 & 0.01 & 0.11 & 0.04 & 0.1 & 1.32 \\
\hline 0.042 & 1.22 & 1 & 3.85 & 0.05 & & & & & & \\
\hline $\mathrm{S} 2$ & Kokoben (outskirts) & 27 & 7.54 & 488.5 & 0.02 & 0.01 & 0.3 & 0.03 & 0.06 & 0.62 \\
\hline 0.076 & 7.98 & 1 & 59.25 & 0.01 & & & & & & \\
\hline S3 & Kokoben town & 28 & 7.44 & 440.5 & 0.2 & 0.01 & 0.25 & 0.03 & 0.15 & 0.59 \\
\hline 0.014 & 5.6 & 0.01 & 3.34 & 0.01 & & & & & & \\
\hline S4 & Yapesa Krofofro & 28 & 7.42 & 312.5 & 0.03 & 0.01 & 0.19 & 0.02 & 0.08 & 0.36 \\
\hline 0.008 & 4.84 & 1 & 0.97 & 0.01 & & & & & & \\
\hline S5 & Bodwesango town B & 28.5 & 6.02 & 146.5 & 0.01 & 0.01 & 0.17 & 0.07 & 0.06 & 1.16 \\
\hline 0.008 & 5.01 & 1 & 0.16 & 0.01 & & & & & & \\
\hline \multirow[t]{2}{*}{ WHO } & & 25 & $6.5-8.5$ & 1500 & 0.05 & 10.00 & 10.05 & 0.3 & 0.3 & 0.00550 \\
\hline & 250 & 0.05 & & & & & & & & \\
\hline
\end{tabular}

TABle 2

Range and mean values of parameters measured

\begin{tabular}{|c|c|c|c|c|c|}
\hline \multirow[t]{2}{*}{ Parameter } & \multirow{2}{*}{\multicolumn{3}{|c|}{ Waterbody }} & \multirow{2}{*}{\multicolumn{2}{|c|}{ Well }} \\
\hline & & & & & \\
\hline \multicolumn{6}{|l|}{ Stream } \\
\hline \multirow[t]{2}{*}{ Range of values } & Dry & Wet & Dry & Wet & Dry \\
\hline & Wet & & & & \\
\hline \multirow[t]{2}{*}{$p \mathrm{H}$} & $4.67-7.01$ & $4.29-7.24$ & $4.09-6.57$ & $4.67-6.62$ & $6.23-7.36$ \\
\hline & $5.81-7.74$ & & & & \\
\hline \multirow[t]{2}{*}{ Conductivity $(\mu \mathrm{S} / \mathrm{cm})$} & $140-840$ & $133-1553$ & $120-780$ & $96-1067$ & $182-540$ \\
\hline & $133-498$ & & & & \\
\hline \multirow[t]{2}{*}{$\mathrm{Ni}$} & $<0.01$ & $<0.01-0.14$ & $<0.01$ & $0.01-0.13$ & $<0.01$ \\
\hline & $0.01-0.08$ & & & & \\
\hline \multirow[t]{2}{*}{$\mathrm{Cu}$} & $<0.01-0.04$ & $<0.01-0.11$ & $<0.01-0.07$ & $<0.01-0.04$ & $<0.01$ \\
\hline & $<0.01-0.01$ & & & & \\
\hline \multirow[t]{2}{*}{$\mathrm{Hg}$} & $<0.001-0.52$ & $<0.001-0.5$ & $<0.001-0.6$ & $<0.001-0.3$ & $0.19-1.33$ \\
\hline & $0.1-0.7$ & & & & \\
\hline \multirow[t]{2}{*}{$\mathrm{Pb}$} & $<0.01-2.02$ & $<0.01-0.06$ & $<0.01-0.05$ & $<0.01-0.04$ & 0.03-0.09 \\
\hline & $<0.01-0.05$ & & & & \\
\hline $\mathrm{Mn}$ & $<0.01-1.09$ & $<0.01-0.68$ & $<0.01-0.42$ & $<0.01-0.33$ & $0.07-0.3$ \\
\hline
\end{tabular}




\begin{tabular}{|c|c|c|c|c|c|}
\hline & $0.01-0.03$ & & & & \\
\hline $\mathrm{Fe}$ & $\begin{array}{l}<0.01-2.12 \\
0.2-2.36\end{array}$ & $<0.01-0.63$ & $0.05-1.52$ & $<0.01-0.39$ & $0.28-2.12$ \\
\hline $\mathrm{Cr}$ & $\begin{array}{l}<0.01-0.068 \\
\mathrm{a}\end{array}$ & $\mathrm{a}$ & $0.01-0.083$ & $\mathrm{a}$ & $<0.01-0.071$ \\
\hline $\mathrm{Cd}$ & $\begin{array}{l}<0.001-0.024 \\
<0.001-0.076\end{array}$ & $<0.001-0.01$ & $<0.001-0.012$ & $<0.001-0.016$ & $<0.001-0.013$ \\
\hline $\mathrm{Zn}$ & $\begin{array}{l}\mathrm{a} \\
0.01-1.00\end{array}$ & $0.01-1.00$ & 2.01-12.59 & $0.01-1.00$ & $\mathrm{a}$ \\
\hline $\mathrm{Mg}$ & $\begin{array}{l}1.12-14.03 \\
0.15-13.8\end{array}$ & $0.3-11.1$ & $\mathrm{a}$ & $0.27-9.01$ & $1.00-2.16$ \\
\hline $\mathrm{Ca}$ & $0.41-52.14$ & $\mathrm{a}$ & $0.58-9.13$ & $\mathrm{a}$ & $0.46-59.25$ \\
\hline $\begin{array}{l}\text { Mean of values } \\
p \mathrm{H}\end{array}$ & $\begin{array}{l}6.01 \\
7.18\end{array}$ & 5.97 & 5.5 & 5.6 & 7.04 \\
\hline Conductivity $(\mu \mathrm{S} / \mathrm{cm})$ & $\begin{array}{l}398 \\
299\end{array}$ & 462 & 377.5 & 407 & 348 \\
\hline
\end{tabular}

a: Not determined

Concentrations of trace metals in $\mathrm{mg} / \mathrm{l}$

\section{$\mathrm{pH}$ and conductivity}

The $p \mathrm{H}$ values of boreholes from the study area ranged from 4.67 to 7.01 with a mean value of 6.01 during the dry season, while the wet season recorded $p \mathrm{H}$ ranging from 4.29 to 7.24 with a mean value of 5.97. The $p \mathrm{H}$ values of the wells from the study area ranged from 4.09 to 6.75 with a mean value of 5.5 during the dry season while the wet season recorded $p \mathrm{H}$ ranging from 4.67 to 6.62 with a mean value of 5.6. The stream sources investigated from the study area recorded $p \mathrm{H}$ values ranging from 6.23 to 7.36 with a mean value of 7.04 during the dry season while the wet season recorded $p \mathrm{H}$ ranging from 5.81 to 7.74 with a mean value of 7.18.

The conductivity values of boreholes from the study area ranged from 140 to $840 \mu \mathrm{S} / \mathrm{cm}$ with a mean value of $398 \mu \mathrm{S} / \mathrm{cm}$ during the dry season while the wet season conductivity values ranged from 133 to $1553 \mu \mathrm{S} / \mathrm{cm}$ with a mean value of $462 \mu \mathrm{S} / \mathrm{cm}$. The conductivity values of wells from the study area ranged from 120 to $780 \mu \mathrm{S} / \mathrm{cm}$ with a mean value of $377.5 \mu \mathrm{S} / \mathrm{cm}$ during the dry season while the wet season conductivity values ranged from 96 to $1067 \mu \mathrm{S} / \mathrm{cm}$ with a mean value of $407 \mu \mathrm{S} / \mathrm{cm}$. The stream sources sampled from the study area recorded conductivity values ranging from 180 to $540 \mu \mathrm{S} / \mathrm{cm}$ with a mean value of $348 \mu \mathrm{S} / \mathrm{cm}$ during the dry season while the wet season recorded conductivity values ranging from 113 to $498 \mu \mathrm{S} / \mathrm{cm}$ with a mean value of $299 \mu \mathrm{S} / \mathrm{cm}$.

\section{Trace metals}

Trace metals considered in this study included $\mathrm{Ni}, \mathrm{Cu}, \mathrm{Hg}, \mathrm{Pb}, \mathrm{Mn}, \mathrm{Fe}, \mathrm{Cr}, \mathrm{Zn}, \mathrm{Cd}, \mathrm{Mg}$ and $\mathrm{Ca}$. The results of the trace metal concentrations for boreholes during the dry season (Table 3) showed that $\mathrm{Ni}$ concen-trations in the study area were all below the background levels $(<0.01$ $\mathrm{mg} / \mathrm{l}), \mathrm{Cu}$ concentrations ranged from $<0.01$ to $0.04 \mathrm{mg} / \mathrm{l}, \mathrm{Hg}$ concentrations ranged from < 0.001 to $0.52 \mathrm{mg} / \mathrm{l}, \mathrm{Pb}$ concentrations ranged from $<0.01$ to $2.02 \mathrm{mg} / \mathrm{l}$, Mn concentrations ranged from $<$ 0.01 to $1.09 \mathrm{mg} / \mathrm{l}$, Fe concentrations ranged from $<0.01$ to $2.12 \mathrm{mg} / \mathrm{l}, \mathrm{Cr}$ concentrations ranged from $<0.01$ to $0.068 \mathrm{mg} / \mathrm{l}, \mathrm{Cd}$ concentrations ranged from $<0.001$ to $0.024 \mathrm{mg} / 1$ and $\mathrm{Mg}$ concentrations ranged from 1.12 to $14.03 \mathrm{mg} / \mathrm{l}$. 


$\begin{array}{lcccc}\text { Element } & \begin{array}{c}\text { Borehole water } \\ (\mathrm{mg} / \mathrm{l})\end{array} & \begin{array}{c}\text { Well water } \\ (\mathrm{mg} / \mathrm{l})\end{array} & \begin{array}{c}\text { Stream water } \\ (\mathrm{mg} / \mathrm{l})\end{array} & \begin{array}{c}\text { WHO guideline } \\ \mathrm{mg} / \mathrm{l}\end{array} \\ \mathrm{Hg} & 0.19 & 0.24 & 0.66 & \\ \mathrm{~Pb} & 0.57 & \mathrm{ND} & 0.09 & 0.001 \\ \mathrm{Mn} & 0.62 & 0.42 & \mathrm{ND} & 0.05 \\ \mathrm{Fe} & 0.99 & 0.8 & 1.08 & 0.3 \\ \mathrm{Cr} & 0.12 & 0.07 & 0.07 & 0.3 \\ \mathrm{Cd} & 0.01 & 0.009 & 0.009 & 0.05 \\ & & & & 0.005\end{array}$

$\mathrm{ND}=$ Not determined

Trace metal concentrations for boreholes during the wet season (Table 4) showed that $\mathrm{Ni}$ concentrations ranged from $<0.01$ to $0.14 \mathrm{mg} / \mathrm{l}, \mathrm{Cu}$ concentrations ranged from $<0.01$ to 0.11 $\mathrm{mg} / \mathrm{l}, \mathrm{Hg}$ concentrations ranged from $<0.001$ to $0.5 \mathrm{mg} / \mathrm{l}, \mathrm{Pb}$ concentrations ranged from $<0.01$ to $0.06 \mathrm{mg} / \mathrm{l}$, Mn concentrations ranged from $<0.01$ to $0.68 \mathrm{mg} / \mathrm{l}$, Fe concentrations ranged from $<0.01$ to $0.63 \mathrm{mg} / \mathrm{l}, \mathrm{Cd}$ concentrations ranged from $<0.001$ to $0.01 \mathrm{mg} / \mathrm{l}, \mathrm{Zn}$ concentrations ranged from 0.01 to $1.00 \mathrm{mg} / \mathrm{l}, \mathrm{Mg}$ concentrations ranged from 0.3 to $11.1 \mathrm{mg} / \mathrm{l}$ and $\mathrm{Ca}$ concentrations ranged from 0.41 to $52.14 \mathrm{mg} / \mathrm{l}$.

TABLE 4

Mean values of trace metals with concentrations exceeding WHO guideline value (wet season)

$\begin{array}{lcccc}\text { Element } & \begin{array}{c}\text { Borehole water } \\ (\mathrm{mg} / \mathrm{l})\end{array} & \begin{array}{c}\text { Well water } \\ (\mathrm{mg} / \mathrm{l})\end{array} & \begin{array}{c}\text { Stream water } \\ (\mathrm{mg} / \mathrm{l})\end{array} & \begin{array}{c}\text { WHO guideline } \\ (\mathrm{mg} / \mathrm{l})\end{array} \\ \mathrm{Ni} & 0.9 & 0.09 & 0.08 & 0.05 \\ \mathrm{Hg} & 0.22 & 0.3 & 0.32 & 0.001 \\ \mathrm{~Pb} & 0.06 & \mathrm{ND} & \mathrm{ND} & 0.05 \\ \mathrm{Mn} & 0.43 & 0.33 & \mathrm{ND} & 0.3 \\ \mathrm{Fe} & 0.56 & 0.39 & 1.15 & 0.3 \\ \mathrm{Cd} & 0.01 & 0.013 & 0.044 & 0.005\end{array}$

$\mathrm{ND}=$ Not determined

Trace metal concentra-tions for wells during the dry season showed that Ni concentrations in the study area were all below the background levels $(<0.01 \mathrm{mg} / \mathrm{l}), \mathrm{Cu}$ concentra-tions ranged from $<0.01$ to $0.07 \mathrm{mg} / \mathrm{l}, \mathrm{Hg}$ concentrations ranged from $<0.001$ to $0.6 \mathrm{mg} / \mathrm{l}, \mathrm{Pb}$ concentrations ranged from $<0.01$ to $0.05 \mathrm{mg} / \mathrm{l}$, Mn concentrations ranged from $<0.01$ to $0.42 \mathrm{mg} / \mathrm{l}$, Fe concentrations ranged from 0.05 to $1.52 \mathrm{mg} / \mathrm{l}, \mathrm{Cr}$ concentrations ranged from $<0.01$ to $0.083 \mathrm{mg} / \mathrm{l}$, Cd concentrations ranged from $<0.001$ to $0.012 \mathrm{mg} / 1$ and $\mathrm{Mg}$ concentrations ranged from 2.01 to $12.59 \mathrm{mg} / \mathrm{l}$.

Trace metal concentrations for wells during the wet season showed that $\mathrm{Ni}$ concentrations ranged from 0.01 to $0.13 \mathrm{mg} / \mathrm{l}, \mathrm{Cu}$ concentrations ranged from $<0.01$ to $0.04 \mathrm{mg} / \mathrm{l}, \mathrm{Hg}$ concentrations ranged from $<0.001$ to $0.3 \mathrm{mg} / \mathrm{l}, \mathrm{Pb}$ concentrations ranged from $<0.01$ to 0.04 $\mathrm{mg} / \mathrm{l}, \mathrm{Mn}$ concentrations ranged from $<0.01$ to $0.33 \mathrm{mg} / \mathrm{l}$, Fe concentrations ranged from $<0.01$ to $0.39 \mathrm{mg} / \mathrm{l}, \mathrm{Cd}$ concentrations ranged from $<0.001$ to $0.016 \mathrm{mg} / \mathrm{l}, \mathrm{Zn}$ concentrations ranged from 0.01 to $1.00 \mathrm{mg} / \mathrm{l}, \mathrm{Mg}$ concentrations ranged from 0.27 to $9.01 \mathrm{mg} / \mathrm{l}$ and $\mathrm{Ca}$ concentrations ranged from 0.58 to $9.13 \mathrm{mg} / 1$.

Trace metal concentra-tions for streams during the dry season showed that $\mathrm{Ni}$ and $\mathrm{Cu}$ concentrations in the study area were all below the background levels of $<0.01 \mathrm{mg} / 1$ and $<0.01$ $\mathrm{mg} / \mathrm{l}$, respectively, $\mathrm{Hg}$ concentrations ranged from 0.19 to $1.33 \mathrm{mg} / \mathrm{l}, \mathrm{Pb}$ concentrations ranged from 0.03 to $0.09 \mathrm{mg} / \mathrm{l}, \mathrm{Mn}$ concentrations ranged from 0.07 to $0.3 \mathrm{mg} / \mathrm{l}$, Fe concentrations ranged from 0.28 to $2.12 \mathrm{mg} / \mathrm{l}, \mathrm{Cr}$ concentrations ranged from $<0.01$ to $0.071 \mathrm{mg} / \mathrm{l}, \mathrm{Cd}$ 
concentrations ranged from $<0.001$ to $0.013 \mathrm{mg} / \mathrm{l}$ and $\mathrm{Mg}$ concentrations ranged from 1.00 to $2.16 \mathrm{mg} / 1$.

Trace metal concentrations for streams during the wet season showed that Ni concentrations ranged from 0.01 to $0.08 \mathrm{mg} / \mathrm{l}, \mathrm{Cu}$ concentrations ranged from $<0.01$ to $0.01 \mathrm{mg} / \mathrm{l}, \mathrm{Hg}$ concentrations ranged from 0.1 to $0.7 \mathrm{mg} / \mathrm{l}, \mathrm{Pb}$ concentrations ranged from $<0.01$ to $0.05 \mathrm{mg} / \mathrm{l}$, Mn concentrations ranged from 0.01 to $0.03 \mathrm{mg} / \mathrm{l}, \mathrm{Fe}$ concentrations ranged from 0.2 to 2.36 $\mathrm{mg} / \mathrm{l}, \mathrm{Cd}$ concentrations ranged from $<0.001$ to $0.076 \mathrm{mg} / \mathrm{l}, \mathrm{Zn}$ concentrations ranged from 0.01 to $1.00 \mathrm{mg} / \mathrm{l}, \mathrm{Mg}$ concentrations ranged from 0.15 to $13.8 \mathrm{mg} / \mathrm{l}$ and $\mathrm{Ca}$ concen-trations ranged from 0.46 to $59.25 \mathrm{mg} / 1$.

\section{$\mathrm{pH}$ and conductivity}

\section{Discussion}

The differences in $p \mathrm{H}$ between ground (boreholes and wells) and surface (streams) waters may be due to the influence of aquifer materials on the chemistry of the ground waters. This is due to the fact that the presence of sulphides and carbonaceous matter in the ore formations means that natural geochemical and biochemical degradation (oxidation) of these rocks may occur, when in contact with oxygen gas containing water, leading to increased hydrogen ion activity in the waters with which they are in contact. The chemistry of the sulphide reaction is very complex but can be simplified as follows (Edwards, 1981):

$$
\begin{aligned}
& \mathrm{FeS}_{2(\mathrm{~s})}+\mathrm{H}_{2} \mathrm{O}_{(\mathrm{aq})}+3.5 \mathrm{O}_{2(\mathrm{~g})}=\mathrm{FeSO}_{4(\mathrm{aq})}+\mathrm{H}_{2} \mathrm{SO}_{4(\mathrm{aq})} \\
& \mathrm{H}_{2} \mathrm{SO}_{4(\mathrm{aq})}=2 \mathrm{H}^{+}{ }_{(\mathrm{aq})}+2 \mathrm{SO}_{4^{-}(\mathrm{aq})}
\end{aligned}
$$

Similarly, the reactions of the carbonaceous matter in the water can be presented as follows (Kuyucak Lyew, St-Germain and Wheeland, 1991):

$$
\begin{aligned}
& \mathrm{C}_{\text {(org) }} \mathrm{CO}_{2(\mathrm{~g})} \\
& \mathrm{H}_{2} \mathrm{O}_{(\mathrm{aq})}+\mathrm{CO}_{2(\mathrm{~g})}=\mathrm{H}^{+}{ }_{(\mathrm{aq})}+\mathrm{HCO}_{3^{-}(\mathrm{aq})} \\
& \mathrm{HCO}_{3_{-(\mathrm{aq})}}=\mathrm{H}^{+}{ }_{(\mathrm{aq})}+\mathrm{CO}_{3}{ }_{(\mathrm{aq})}^{2-}
\end{aligned}
$$

Consequently, the hydrogen ion activity of the waters with which the minerals are in contact is increased. However, surface water $p \mathrm{H}$ values fell within 6-9 $p \mathrm{H}$ range of natural waters (Stumn $\&$ Morgan, 1981) except at Bodwesango (5.81 $\mathrm{pH}$ units). Bio-oxidation of sulphur-containing materials may also take place in tailing dumps in abandoned mine areas, and acid mine drainage in exposed workings, as well as in the aquifer may lead to the release of hydrogen ions into the aqueous environment and, therefore, increase the acidity of surface water and ground waters.

Acidity can also be caused by the dissolution of carbon dioxide in the water. This is presented in the equation as follows:

$$
\mathrm{CO}_{2(\mathrm{~g})}+\mathrm{H}_{2} \mathrm{O}_{(\mathrm{aq})}=\mathrm{H}_{(\mathrm{aq})}^{+}+\mathrm{HCO}_{3_{(}^{-}}
$$

Low acidity in the water bodies may, therefore, be attributed either to mining activity (effluent discharge or acid mine drainage), or to natural processes especially in areas where no mining operation is taking place. In the case of the differences in the conductivity values, it may be inferred that, due to the natural geochemical and biochemical degradation processes taking place 
in the aquifer, the waters in contact with these aquifers are likely to contain much higher concentrations of dissolved ions and, therefore, more mineralized than the surface waters. Analysis of the wet and dry season values reveals differences in values (Table 2). These disparities may mainly be due to dilution.

Correlation analyses of the parameters using both wet and dry season data revealed expected processes-based relationship between $\mathrm{Mg}^{2+}$ and $\mathrm{Ca}^{2+}(r=0.76)$, derived mainly from the geochemical and biochemical processes within the aquifer. This may be due to mineralogical influence. However, there were also other strong linear relationships between trace metals, such as that between $\mathrm{Mn}^{2+}$ and $\mathrm{Ca}^{2+}(r=0.63) ; \mathrm{Fe}^{2+}$ and $\mathrm{Ca}^{2+}(r=0.61) ; \mathrm{Fe}^{2+}$ and $\mathrm{Mg}^{2+}(r=0.51)$; $\mathrm{Mg}^{2+}$ and $\mathrm{Mn}^{2+}(r=0.61)$ during the wet season (Table 5), and between $\mathrm{Mg}^{2+}$ and $\mathrm{Mn}^{2+}(r=$ $0.72) ; \mathrm{Hg}^{2+}$ and $\mathrm{Mn}^{2+}(r=0.61) ; \mathrm{Hg}^{2+}$ and $\mathrm{Mg}^{2+}(r=0.50)$ during the dry season (Table 6) that are not normally expected to be linked in terms of processes.

TABLE 5

Correlation matrix showing r-values of water quality parameters in the study area (wet season).

$\begin{array}{lllllllll}T E M P & \mathrm{PH} & \mathrm{COND} & \mathrm{Ni} & \mathrm{Cu} & \mathrm{Hg} & \mathrm{Pb} & \mathrm{Mn} & \mathrm{Fe} \\ \mathrm{Cd} & \mathrm{Mg} & \mathrm{Zn} & \mathrm{Ca} & & & & & \end{array}$

\begin{tabular}{|c|c|c|c|c|c|c|c|c|c|}
\hline TEMP & 1 & & & & & & & & \\
\hline $\mathrm{PH}$ & -0.15 & 1 & & & & & & & \\
\hline COND & 0.00 & 0.15 & 1 & & & & & & \\
\hline $\mathrm{Ni}$ & -0.01 & 0.03 & 0.35 & 1 & & & & & \\
\hline $\mathrm{Cu}$ & 0.26 & $-0.60^{\vee}$ & -0.06 & 0.26 & 1 & & & & \\
\hline $\mathrm{Hg}$ & -0.08 & -0.10 & 0.01 & 0.03 & -0.14 & 1 & & & \\
\hline $\mathrm{Pb}$ & -0.10 & -0.11 & -0.11 & 0.16 & 0.29 & -0.01 & 1 & & \\
\hline $\mathrm{Mn}$ & -0.26 & 0.10 & 0.24 & 0.36 & -0.13 & 0.04 & 0.16 & 1 & \\
\hline $\mathrm{Fe}$ & $-0.39 *$ & 0.36 & 0.06 & 0.26 & -0.20 & 0.08 & -0.20 & 0.21 & 1 \\
\hline $\mathrm{Cd}$ & 0.05 & 0.00 & 0.19 & -0.05 & -0.17 & -0.15 & 0.01 & -0.08 & 0.151 \\
\hline $\mathrm{Mg}$ & $-0.48^{*}$ & 0.20 & 0.33 & $0.39 *$ & -0.17 & 0.14 & 0.12 & $0.61^{\vee}$ & $0.51^{\bullet} 0$ \\
\hline .10 & 1 & & & & & & & & \\
\hline $\mathrm{Zn}$ & -0.18 & $0.50^{*}$ & -0.10 & -0.10 & -0.24 & $-0.40^{*}$ & -0.35 & -0.22 & 0.190 \\
\hline .10 & -0.07 & 1 & & & & & & & \\
\hline $\mathrm{Ca}$ & -0.29 & 0.34 & 0.29 & 0.36 & -0.12 & 0.17 & 0.27 & $0.63^{\circ}$ & $0.61^{\circ} 0$ \\
\hline .21 & $0.76^{\bullet}$ & 0.00 & 1 & & & & & & \\
\hline
\end{tabular}

$* \mathrm{p} \leq 0.05 ; \vee \mathrm{p} \leq 0.01 ; \bullet \mathrm{p} \leq 0.001$.

TABLE 6

Correlation matrix showing $r$-values of water quality parameters in the study area (dry season)

$\begin{array}{lllllllll}T E M P & p H & C O N D . & C u & H g & P b & M n & F e & C r \\ C d & M g & & & & & & \end{array}$

TEMP $\quad 1$

$\mathrm{pH} \quad-0.03 \quad 1$ 


\begin{tabular}{|c|c|c|c|c|c|c|c|c|c|}
\hline COND. & 0.13 & 0.24 & 1 & & & & & & \\
\hline $\mathrm{Cu}$ & -0.09 & -0.14 & -0.03 & 1 & & & & & \\
\hline $\mathrm{Hg}$ & 0.21 & 0.14 & $0.64^{\mathrm{fe}}$ & -0.21 & 1 & & & & \\
\hline $\mathrm{Pb}$ & -0.21 & 0.09 & -0.07 & -0.17 & 0.18 & 1 & & & \\
\hline Mn & 0.00 & 0.18 & $0.53^{\text {e\& }}$ & -0.03 & $0.61^{\text {e\& }}$ & 0.27 & 1 & & \\
\hline $\mathrm{Fe}$ & -0.17 & -0.08 & 0.31 & -0.18 & -0.12 & -0.20 & -0.07 & 1 & \\
\hline $\mathrm{Cr}$ & -0.25 & -0.32 & -0.31 & -0.09 & -0.05 & $0.45^{*}$ & -0.21 & -0.09 & 1 \\
\hline $\mathrm{Cd}$ & 0.35 & $0.57^{\mathrm{e \&}}$ & 0.17 & -0.20 & 0.08 & 0.27 & -0.07 & -0.10 & -0 \\
\hline .19 & 1 & & & & & & & & \\
\hline $\mathrm{Mg}$ & 0.03 & $0.56^{\text {e\& }}$ & $0.68^{\mathrm{fe}}$ & -0.06 & $0.50 *$ & 0.21 & $0.72^{\mathrm{fe}}$ & -0.17 & -0 \\
\hline .20 & 0.35 & 1 & & & & & & & \\
\hline
\end{tabular}

\section{Trace metals}

Iron. The major minerals found in igneous rocks; amphiboles, ferromagnesian micas, ferrous sulphide $(\mathrm{FeS})$, ferric sulphide or iron pyrite $\left(\mathrm{FeS}_{2}\right)$, and magnetite $\left(\mathrm{Fe}_{3} \mathrm{O}_{4}\right)$ form the natural sources of iron in ground water (Todd, 1980). According to the geology of the Ashanti gold belt (Dzigbordi-Adjimah, 1991), rock mineral types present include arsenopyrite (FeAsS), magnetite, pyrite $\left(\mathrm{FeS}_{2}\right)$, chalcopyrite $\left(\mathrm{CuFeS}_{2}\right)$, marcasite $\left(\mathrm{FeS}_{2}\right)$, sphalerite $(\mathrm{ZnS})$, bornite $\left(\mathrm{CuFeS}_{4}\right)$, galena $(\mathrm{PbS})$ and iron-rich carbonates. The presence of these minerals in the Ashanti gold belt are major natural sources of iron in ground water. It may, therefore, be inferred that the relatively high iron found in the samples (Tables 3 and 4) may be due to the mineral-water interactions and oxidation-reduction reactions taking place in such systems.

Several factors influence iron concen-tration in water. One of these factors is the oxygen content of water. When oxygen is dissolved in water, a potential $\left(\mathrm{E}^{\mathrm{O}}\right)$ of $+1.23 \mathrm{~V}$ is generated as presented in the following equation:

$$
\mathrm{O}_{2(\mathrm{~g})}+4 \mathrm{H}^{+}+4 \mathrm{e}^{-}=2 \mathrm{H}_{2} \mathrm{O} \mathrm{E}^{\mathrm{O}}=+1.23 \mathrm{~V}
$$

The electrons taken up in the reduction equation above may be derived from a coupled oxidation reaction such as (Drost-Hansen, 1967):

$$
\mathrm{FeS}_{2(\mathrm{~S})}=\mathrm{Fe}^{2+}+\mathrm{S}^{2-}+2 \mathrm{e}^{-}
$$

Surface water bodies (streams) in the study area were most affected by Fe contamina-tion during both dry $((0.28-2.21 \mathrm{mg} / \mathrm{l})$ and wet $(0.2-2.36 \mathrm{mg} / \mathrm{l})$ seasons. This may be due to the $p H$ content ( $p \mathrm{H}$ range 5.81-7.74) of the surface waters in the study area, since in oxygenated surface waters $(p \mathrm{H} \mathrm{5-8)}$, typical concentrations of total iron are around $0.05-2 \mathrm{mg} / 1$ (U.S. Environmental Protection Agency, 1980). 
Boreholes in the study area were also affected by Fe contamination both during the dry $(0.99$ $\mathrm{mg} / \mathrm{l})$ and wet $(0.56 \mathrm{mg} / \mathrm{l})$ seasons, whilst wells were the least affected by Fe contamination during both dry $(0.8 \mathrm{mg} / \mathrm{l})$ and wet $(0.39 \mathrm{mg} / \mathrm{l})$ seasons. Fig. 2 and 3 presents the mean values of trace metals with concentrations exceeding WHO guideline value of $0.3 \mu \mathrm{g} / \mathrm{l}$ for dry and wet seasons, respectively.

Cadmium. Cd concentrations in unpolluted natural waters are usually below $1 \mu \mathrm{g} / \mathrm{l}$. The estimated lethal oral dose for humans is $350-3500 \mathrm{mg}$ of $\mathrm{Cd}$; a dose of $3 \mathrm{mg}$ of Cd has no effects on adults (WHO, 1996). Wells and stream sources in the study area had the same mean values $(0.009$ $\mathrm{mg} / \mathrm{l}$ ) of trace metals with concentrations exceeding the WHO guideline value of $0.005 \mathrm{mg} / \mathrm{l}$ during the dry season. However, during the wet season, stream sources recorded a higher mean $\mathrm{Cd}$ value $(0.044 \mathrm{mg} / \mathrm{l})$. Boreholes were the least affected by Cd contamination, with a mean value of $0.01 \mathrm{mg} / 1$ during both wet and dry seasons.

$\mathrm{Cd}$ is chemically similar to Zinc and occurs naturally with $\mathrm{Zn}$ and $\mathrm{Pb}$ in sulphide ores (e.g. $\mathrm{ZnS}$ and $\mathrm{PbS}$ ). These ores constitute the mineral geology of the Ashanti gold belt, therefore, during natural geochemical and biochemical degradation processes $\mathrm{Cd}$ may possibly be substituted for $\mathrm{Zn}$ or $\mathrm{Pb}$. It may, therefore, be inferred that the relatively high $\mathrm{Cd}$ found in the samples may be due to the mineral-water interactions. Tables 3 and 4 present histogram of trace metals with concentra-tions exceeding WHO guideline value of $0.005 \mathrm{mg} / \mathrm{l}$ for dry and wet seasons, respectively.

Nickel. In aqueous solution, nickel occurs mostly as the green hexa-aquanickel (II) ion, $\mathrm{Ni}$ $\left(\mathrm{H}_{2} 0\right)_{6}{ }^{2+}$. The nickel ion content of ground water may increase as a result of the oxidation of natural nickel containing ferrosulphate deposits (WHO, 1996). Oxidation can occur if the ground water table is lowered or if nitrate has leached from the soil. Nickel concentrations in ground water around the world are normally $<20 \mu \mathrm{g} / 1$, although levels up to several hundred micrograms per litre in ground water and drinking water have been reported (WHO, 1996). Leaching from nickel-chromium plated taps and fittings could also be sources of nickel in water.

Nickel levels in all water sources were below the background levels $(<0.001 \mathrm{mg} / \mathrm{l})$ during the dry season. However, during the wet season, nickel concentrations in boreholes were high (mean value $0.9 \mathrm{mg} / \mathrm{l}$ ). Wells and streams recorded mean values of 0.09 and $0.08 \mathrm{mg} / 1$, respectively. The differences in nickel concentrations during the wet and dry seasons could be due to dilution. The slightly high $\mathrm{Ni}$ concentrations measured in the ground waters, especially boreholes, seemed to indicate that it might have resulted from interactions within the aquifer. Fig. 2 and 3 present the mean values of trace metals with concentrations exceeding WHO guideline value of $0.05 \mathrm{mg} / \mathrm{l}$ for wet and dry seasons, respectively.

Mercury. Stream sources were the most affected by mercury contamination during both dry $(0.66 \mathrm{mg} / \mathrm{l}$ mean value) and wet $(0.32 \mathrm{mg} / 1$ mean value) seasons. Wells recorded the next higher mercury contamination with mean value of $0.24 \mathrm{mg} / \mathrm{l}$ for dry season and $0.3 \mathrm{mg} / \mathrm{l}$ for wet season. Boreholes were the least affected by mercury contamination in the study area with mean value of $0.19 \mathrm{mg} / \mathrm{l}$ for dry season and $0.22 \mathrm{mg} / \mathrm{l}$ for wet season. A major source of mercury pollution within the Ashanti gold belt is gold recovery using mercury amalgamation as practiced by smallscale miners. Evidence of this practice could be seen in some areas around Konongo, Odumase and Koniyaw. This, therefore, means that the relatively high concentration of $\mathrm{Hg}$ in the samples may be attributed to gold recovery using mercury amalgamation. Naturally occurring levels of mercury in surface and ground water is $<0.5 \mu \mathrm{g} / \mathrm{l}$. Tables 3 and 4 presents histograms of trace metals with concentrations exceeding WHO guideline value of $0.001 \mathrm{mg} / \mathrm{l}$ for dry and wet seasons, respectively. 


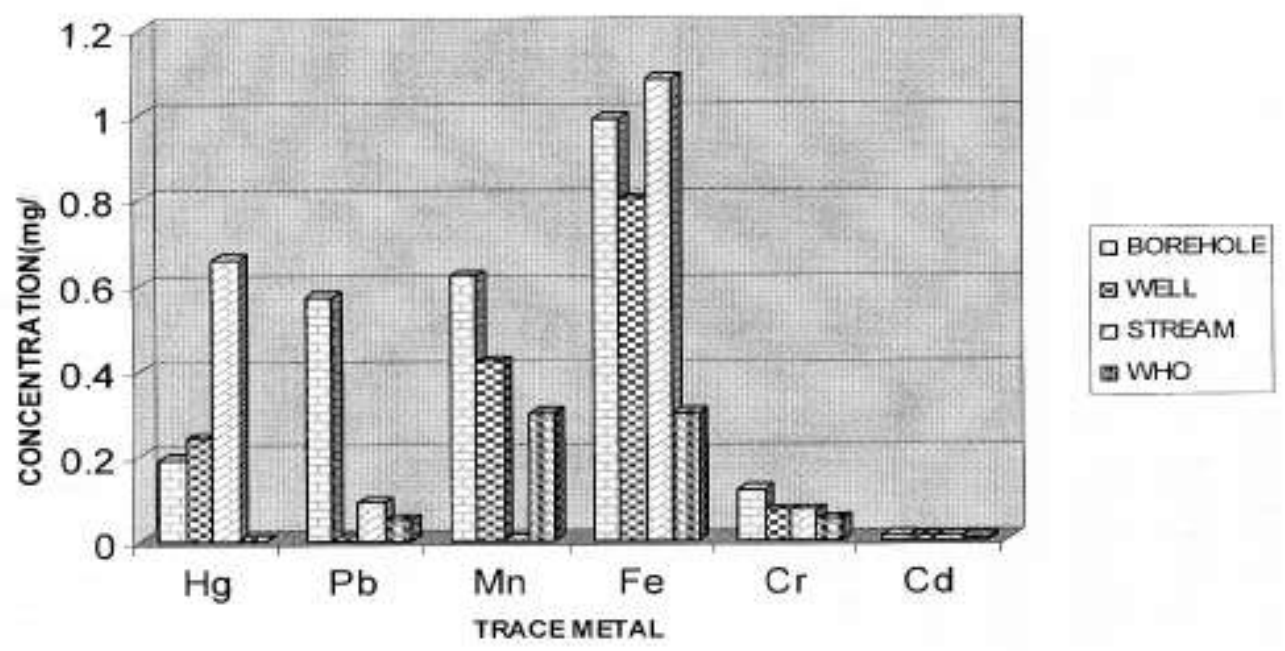

Fig. 3. Histogram of trace metals with concentrations exceeding WHO guideline value (dy season).

Manganese. Generally, $\mathrm{Mn}$ is very abundant in rocks and soils, typically in the form of manganese oxides and hydroxides in association with other metallic cations. At low and neutral $p \mathrm{H}$ values, the predominant dissolved form of manganese is as the divalent cation, $\mathrm{Mn}^{2+}$. Manganese concen-trations $>0.1 \mathrm{mg} / \mathrm{l}$ impart an undesirable taste to drinking water. At about 0.2 $\mathrm{mg} / \mathrm{l}$ manganese concentration, manganese will form coatings on piping that may later tear off as a black precipitate. Concentrations of the order of $0.1-1 \mathrm{mg} / \mathrm{l}$ are common, although in low $p \mathrm{H}$ waters higher concentrations can occur (U.S. Environ-mental Protection Agency, 1980).

Surface water (streams) sources investigated in this study had manganese concentrations below the WHO guideline value $(0.3 \mathrm{mg} / \mathrm{l})$ for both dry and wet seasons. Boreholes sampled in the study area were most affected by manganese contamination during both dry (mean value 0.62 $\mathrm{mg} / \mathrm{l}$ ) and wet (mean value $0.43 \mathrm{mg} / \mathrm{l}$ ) seasons. Well water sources sampled in the study area were also affected by manganese contamination with mean values of 0.42 and $0.33 \mathrm{mg} / \mathrm{l}$ for wet the and dry seasons, respectively. Fig. 2 and 3 present the mean values of trace metals with concentrations exceeding WHO guideline value for wet and dry seasons, respectively.

Lead. Lead is the most common of the heavy elements, accounting for $13 \mathrm{mg} / \mathrm{kg}$ of the earth's crust. More than $80 \%$ of the daily intake of lead is derived from the ingestion of food, dirt and dust. That means that an average of $5 \mu \mathrm{g} / \mathrm{l}$ lead intake from water forms a relatively small proportion of the total daily intake for children and adults but significant for bottle-fed infants. Lead is a possible human carcinogen (evidence inadequate in humans but sufficient in animals) and a cumulative poison and, therefore, any increase in the body burden of lead should be avoided.

It is generally rare to have $\mathrm{Pb}$ concentra-tions outside WHO standard for drinking water. Well water sources investigated in the study area had lead concentrations below the WHO guideline value $(0.05 \mathrm{mg} / \mathrm{l})$ both during the dry and wet seasons. Borehole water sources were the most affected by lead contamination with mean values $0.57 \mathrm{mg} / 1$ and $0.06 \mathrm{mg} / \mathrm{l}$ for dry and wet seasons, respectively. Surface water (stream) sources investigated in the study area had a slightly higher mean value $(0.09 \mathrm{mg} / \mathrm{l})$ during the dry season than the WHO guideline value. Possible reasons that could be attributed to the relatively high lead content in borehole water sources in the study area are the possible dissolution of the rocks through which the water passes, the use of leaded gasoline leading to fallout from exhausts or the constituent minerals of the Ashanti gold 
belt. Fig. 2 and 3 present histograms of trace metals with concentrations exceeding WHO guideline value for wet and dry seasons, respectively.

Chromium. Chromium is widely distributed in the earth's crust. In water, chromium (III) is a positive ion that forms hydroxides and complexes, and is adsorbed at relatively high $p \mathrm{H}$ values. The ratio of chromium (III) to chromium (VI) varies widely in surface water. In general, chromium (VI) salts are more soluble than those of chromium (III), thus, chromium (VI) is, relatively mobile. The daily chromium requirement for adults is estimated to be $0.5-2 \mu \mathrm{g}$ of absorbable chromium (III). That equals to approximately $2-8 \mu \mathrm{g}$ of chromium (III) per day since only about $25 \%$ can be absorbed. The chromium concentrations in ground water is generally low $(<1 \mu \mathrm{g} / \mathrm{l})$. The natural total chromium content of surface water is approximately $0.5-2.0 \mu \mathrm{g} / \mathrm{l}$ and the dissolved chromium content $0.02-0.3 \mu \mathrm{g} / \mathrm{l}$. Most surface water contain $1-10 \mu \mathrm{g} / \mathrm{l}$ of chromium. In general, the chromium content of surface water reflects the extent of industrial activity.

Chromium analyses was done only during the dry season and the results showed that boreholes were the most affected by chromium contamination with a mean value of $0.12 \mathrm{mg} / \mathrm{l}$, whilst well and stream water sources recorded the same mean value of $0.07 \mathrm{mg} / \mathrm{l}$ slightly above the WHO guideline value $0.05 \mathrm{mg} / \mathrm{l}$. The relatively high $\mathrm{Cr}$ content in the study area may be due to the presence of $\mathrm{Cr}$ in trace quantities in the minerals found in the Ashanti gold belt. Fig. 3

presents the mean values of chromium with concentrations exceeding WHO guideline value for the dry season.

Other trace metals. Other trace metals considered in this study included $\mathrm{Mg}, \mathrm{Zn}, \mathrm{Cu}$ and $\mathrm{Ca}$. The results of the analyses revealed that the concentrations of these trace metals in the water bodies investigated during both wet and dry seasons in the study area were all below the WHO threshold.

\section{Conclusion}

Ground water had lower $p \mathrm{H}$, ranging from 4.09 to $7.24 p \mathrm{H}$ units than surface water, ranging from 5.81 to $7.74 p \mathrm{H}$ units. The $p \mathrm{H}$ of many of the wells and boreholes were below 6 both in the dry and wet seasons. This may be due to natural geochemical and biochemical processes within the aquifers. Ground water had higher concentrations of dissolved ions (ranging from $96 \mu \mathrm{S} / \mathrm{cm}$ to $1553 \mu \mathrm{S} / \mathrm{cm}$ ) and are, therefore, more mineralized than surface waters (ranging from $133 \mu \mathrm{S} / \mathrm{cm}$ to $540 \mu \mathrm{S} / \mathrm{cm}) . \mathrm{Mg}, \mathrm{Zn}, \mathrm{Cu}$ and $\mathrm{Ca}$ concentrations were all below the WHO guideline values throughout the study.

Surface water bodies (streams) in the study area were found to be the most affected by $\mathrm{Fe}, \mathrm{Cd}$ and $\mathrm{Hg}$ contamination. Iron contamination may be due to the fact that surface waters are oxygenated $(p \mathrm{H} \mathrm{5-8)}$, cadmium contamination may be due to its natural occurrence with $\mathrm{Zn}$ and Pd in sulphide ores of the Ashanti gold belt, and mercury contamination may be due to gold recovery using mercury amalgamation by small-scale miners.

Ground water sources in the study area were established to be the most affected by $\mathrm{Ni}, \mathrm{Mn}, \mathrm{Pb}$ and $\mathrm{Cr}$ contamination. Ni contamination may be due to interactions within the aquifer $\mathrm{Mn}$ contamination may be due to natural geochemical and biochemical processes within the aquifer. $\mathrm{Pb}$ contamination may be due to the possible dissolution of the rocks through which the water passes. The use of leaded gasoline leading to fallout from exhausts or the constituent minerals of the Ashanti gold belt, and $\mathrm{Cr}$ contamination may be due to the presence of $\mathrm{Cr}$ in the minerals found in the Ashanti gold belt.

Correlations between the trace metals revealed expected process-based relation-ship between $\mathrm{Mg}^{2+}$ and $\mathrm{Ca}^{2+}(r=0.76)$, derived mainly from the geochemical and biochemical processes within the aquifer. However, there were also other strong linear relationships between trace metals, such as $\mathrm{Fe}^{2+}$ and $\mathrm{Ca}^{2+}(r=0.61) ; \mathrm{Mg}^{2+}$ and $\mathrm{Mn}^{2+}(r=0.72) ; \mathrm{Hg}^{2+}$ and $\mathrm{Mn}^{2+}(r=0.61)$, that are not 
normally expected to be linked in terms of processes. Compared to WHO guideline, all the well and stream water sources had one or more trace metal level outside acceptable limits set by the WHO for drinking water, and only three borehole water samples at Konongo (Abronikrom), Ntronang (Ahenbronoso) and Bomfa (Nkubem) were safe for human consumption.

\section{Acknowledgement}

The study forms part of the comprehensive study of the quality of water bodies within the Ashanti gold belt by the Institute of Mining and Mineral Engineering (IMME) of the Kwame Nkrumah University of Science and Technology, Kumasi, in collaboration with the Noguchi Memorial Institute of Medical Research (NMIMR), University of Ghana, Legon. Facilities provided by all these institutions are gratefully acknowledged.

\section{Referrences}

Afenya P. M. (1976). Studies on the processing of a carbonaceous gold ore. (PhD Thesis.), University of London.

Amanor J. A. (1979). The geology of the Ashanti gold mines and implication for exploration (MSc. Thesis.) University of London.

Amono-Niezer E. H and Amenkor, E. M. K (1993). Determination of total arsenic in environmental samples from Kumasi and Obuasi. Envir. Hlth Perspect. 101(1): 46-49.

Anon. (1999). Private communication with Prof. F. W. Y. Momade. Kwame Nkrumah, University of Science and Technology, Kumasi, Ghana.

Barcelona M., Gibb J. P., Helfrich J. A. and Garske E. E. (1985). Practical guide for groundwater sampling. Ilinois State Water Survey ISWS Contract Report 37.

Boyle R. W. (1959), The geochemistry origin and role of carbon dioxide, sulphur, water and boron in the YellowKnife gold deposits of N.W.T. Canada. Econ. Geol. 54(8): 1506-1524.

Carboo D. and Sarfo-Armah Y. (1997). Arsenic pollution in streams and sediments on the mining industry and the environment, Kumasi, Ghana. pp. 114-119.

Claasen H. C. (1982). Guidelines and techniques for obtaining water samples that accurately represent the quality of an aquifer. U.S. Geological Survey Open File Report 82-1024. 49 pp.

Cooper W. G. (1934). The geology of the Prestea Goldfields. Gold Coast Geological Survey Memoir No. 3.

Drost-Hansen W. (1967). The structure of water and water-solute interaction. Equilibrium concepts in natural water systems. Adv. Chem. Ser. No. 67. American Chemical Society, Washington, DC.

Dzigbordi-Adjimah K (1988). The Genesis of the Birimian Gold Deposits of Ghana. Geological Society of Ghana Proceedings of the International Conference on the Geology of Ghana with special emphasis on Gold. 9th to 10th October, vol.1, 1991, pp. F1-F29.

Dzigbordi-Adjimah K. (1991). Environmental Concerns of Ghana's Gold Booms, Past, Present and Future. Ghana Min. J. 2(1): 21-26.

Edwards R. W. (1981). The impact of coal mining on river ecology, scientific section symposium on mining and water pollution. Institute of Water Engineers and Scientists.

Golow A. A., Schlueter A., Amihere-Mensah S., Granson H. L. K. and Tetteh M. S. (1996). Distribution of arsenic and sulphate in the vicinity of Ashanti Goldmine at Obuasi, Ghana. Bull. Environ. Contam. Toxicol. 56: 703-710.

Hofkes E. H. (1986), Small Community Water Supplies. John Willey and Sons, Chichester, New York, pp. 42-66.

Junner N. R et al (1932). The Geology of Obuasi Goldfields. Gold Coast Geological Survey Memoir No. 2

Junner N. R (1935). Gold in The Gold Coast. Gold Coast Geological survey Memoir No. 4.

Kuyucak N. Lyew D. St-Germain and Wheeland (1991). In situ bacterial treatment of acid mine drainage in open pits. 2nd International Conference on the Abatement of Acidic Drainage. 16-18th September 1991, Montreal, Canada. pp. 335-368.

Mpendazoe F. M. T. (1996). Towards a sustainable small scale gold mining in Tanzania. Proceedings of the First World Mining Environment Congress. Thakur, New Delhi, India,11-14 December, 1995. (B. B. Dhar and D. N. Thakur, ed.), pp. 105-119. AA Balkema, Rotterdam.

Ntiamoah-Agyakwa (1974). Obuasi Gold Deposits of Ghana. (MSc. Thesis.) University of Western Ontario, Canada.

Ntiamoah-Agyakwa (1979). Relationship between gold and manganese mineralization in the Birimian of Ghana, $W$. Afr. Geol. Mag. 116: 342-352.

RT1 ITDG (2001). Small scale mining and sustainable development within the SADC Region, MMSD SOUTHERN AFRICA Report on Research Topic 1. (B. Dreschler, ed.). ITDG. 204 pp. 
Sarkordie P. A., Nyamah D. and Amonoo-Niezer E. H. (1997). Speciation of Arsenic in some Biological Samples from Obuasi and its Surrounding Villages. Procedings of a National Symposium on The Mining Industry and the Environment, Kumasi, Ghana.pp. 147-154.

Stumn W. and Morgan J. J. (1981). Aquatic chemistry. Willey, New York. 780 pp.

Todd D. K (1980). Groundwater hydrology, 2nd edn. John Wiley and Sons, Inc. N.Y, Chichester, Brisbane, Toronto. pp. 271-277.

U.S Environmental Protection Agency (1980). Quality Criteria for Water. U.S EPA, Office of Water. EPA/440/5-80 Series, Washington, DC.

WHO (1996). Guidelines for drinking-water quality, 2nd edn, vol. 2. World Health Organization, Geneva. 